\title{
Cartografía de las resistencias en Mendoza: saberes y estrategias de los movimientos sociales en contextos de pandemia
}

Cartography of resistance in Mendoza. Knowledge and strategies of social movements in pandemic contexts

María Molina Guiñazú

Universidad Nacional de Cuyo - Argentina

Mendoza, Argentina

milagrosmolinag@hotmail.com

Mariana Polti

Universidad Nacional de Cuyo - Argentina

Mendoza, Argentina

marianapolti@gmail.com

Oscar Soto

Universidad Nacional de Cuyo - Argentina

Mendoza, Argentina

osoto@fcp.uncu.edu.ar

\section{RESUMEN}

El presente trabajo se escribe alrededor de dos grandes interrogantes: ¿Qué impacto estaba teniendo la pandemia en sus territorios? y ¿Qué estrategias se daban para enfrentarla? El objetivo principal fue identificar las estrategias que trabajaron distintas organizaciones y movimientos sociales de la provincia de Mendoza (Argentina) para enfrentar la crisis que trajo aparejada la pandemia producida por el COVID-19 durante el periodo que abarcó los meses de marzo a julio del año 2020. Así, inscriptos/as en la tradición de la investigación militante y sus elaboraciones teórico-metodológicas; nos propusimos a través de un dispositivo grupal, en este caso un conversatorio virtual, la generación de un espacio para el intercambio junto a organizaciones político-territoriales locales, como un primer paso para definir los términos del debate. Se trata entonces de un estudio exploratorio- descriptivo en el que intentamos esbozar una cartografía de las resistencias en Mendoza a través de la sistematización del repertorio de acción de los movimientos en torno a las principales problemáticas que se fueron identificando en el intercambio: alimentación, salud, educación y trabajo. Nos interesa con esta investigación, no sólo visibilizar cómo el sostenimiento de la vida de un amplio sector de las clases populares mendocinas ha estado fuertemente vinculado a la intervención político-territorial de las organizaciones; sino también aportar a los procesos de construcción colectiva de conocimientos vinculando universidades y movimientos/ territorios/comunidades.

Palabras clave: Movimiento social; Argentina; autogestión; economía colectiva; pandemia.

\section{ABSTRACT}

This paper was written around two main questions: What impact was the pandemic having on their territories and what strategies were being used to face it? The main objective was to identify the strategies used by different organizations and social movements in the province of Mendoza (Argentina) to face the crisis brought about by the pandemic produced by COVID-19 during the period from March to July 2020. Thus, in the tradition of militant research and its theoretical-methodological elaborations, we proposed through a group device, in this case, a virtual conversation, the generation of a space for exchange with local political-territorial organizations, as a first step to define the terms of the debate. This is an exploratory-descriptive study in which we try to outline cartography of the resistances in Mendoza through the systematization of the repertoire of action of the movements around the main issues that were identified in the exchange: food, health, education, and work. We are interested in this research, not only to make visible how the sustainability of the life of a wide sector of the popular classes in Mendoza has been strongly linked to the political-territorial intervention of the organizations but also to contribute to the processes of collective construction of knowledge linking universities and movements/territories/communities.

Keywords: social movement; Argentina; self-management; collective economy; pandemic. 


\section{INTRODUCCIÓN}

Hace más de un año que "un virus recorre el mundo", trastocando el "normal” funcionamiento de la sociedad global. La pandemia producida por el COVID-19 y el aislamiento que trajo aparejado (Decreto $\mathrm{N}^{\circ}$ 297/20 del Poder Ejecutivo Nacional) ha generado una crisis a escala planetaria. Si bien sus efectos a largo plazo aún no logran ser dimensionados en sentido estricto, su impacto se hace patente en la sociedad en general, y sobre la vida de las clases trabajadoras y precarizadas de manera particular (Grupo de Trabajo CLACSO Crisis y economía mundial, 2020; Kessler y Benza, 2020). El incremento del costo de vida junto con diversas formas de violencias, la creciente vulnerabilidad económica y habitacional, entre tantos otros aspectos, exponen de forma descarnada las desigualdades pre-existentes que la pandemia vino a cristalizar (CEDES, 2020; CEPAL, 2020; Informe H. Senado de la Nación Argentina, 2020; Informe Ministerio de Desarrollo Productivo, 2020; Soto y Caisson, 2020).

Frente a este contexto, se intensifica y se resignifica la disputa por la producción de saberes (Ceceña, 2008) dentro y fuera de las universidades y, como parte de un equipo de investigación mayor. Este trabajo se inscribe en una línea de investigación que venimos construyendo desde el año 2017 dentro del proyecto: "Saberes y territorios. La disputa de sentidos desde los movimientos sociales" perteneciente a la Secretaría de Investigación, Internacionales y Posgrado de la Universidad Nacional de Cuyo -SIIP-UNCUYO- (Molina, 2019) intentamos aportar a la producción de saberes que se involucren y aporten a los problemas que aquejan a movimientos sociales y organizaciones político- territoriales, generando una praxis de investigación que asuma su historicidad y las imbricaciones de poder en las que está inmersa.

Nos reconocemos dentro de una rica trayectoria de producciones teóricas, de innovaciones metodológicas y de experiencias de investigación militante existente a lo largo del tiempo en América Latina. Los gérmenes de esta tradición remiten a prácticas comunitarias y a resistencias negras, indígenas, de mujeres y disidencias desde hace siglos, pero ya en el siglo XX, el pensamiento crítico se fortalece y se consolida en la región, buscando construir formas de conocer la realidad para conseguir transformarla a través de la praxis, rechazando aplicaciones mecanicistas de interpretaciones foráneas (Martinez y Guacheta, 2020). Así, la lucha contra los diferentes tipos de colonialismo, de imperialismo y por la liberación de los pueblos fueron la base para la construcción de un pensamiento crítico que conectase la reflexión intelectual con la actuación política transformadora. De este modo, se erigió un pensamiento comprometido con el cambio social y con las luchas y movimientos sociales concretos; intentado aportar al cuestionamiento de todas las formas de dependencia, explotación y subalternidad; y a la construcción de otros saberes en diálogo, pero también en disputa con diversas modalidades y espacios de saber. Inscriptos/as en esta tradición, algunas nociones básicas que orientan nuestro trabajo son las siguientes.

\section{¿Con quiénes y para qué construimos saberes? El trabajo con organizaciones y movimien- tos populares}

Desde nuestra perspectiva, los movimientos y las organizaciones sociales constituyen espacios de participación política que tiene una particularidad: la de hallarse relacionadas con la experiencia vital de los/as sujetos/as. Es decir, cercanas a la compleja trama de necesidades, subjetividades, afirmaciones culturales y proyectos que los sujetos esgrimen (De Sousa Santos, 2001). Su contexto inmediato es caracterizado por una profunda fragmentación social, generada en la multiplicación de experiencias sociales que se desprenden de la situación del capitalismo avanzado. Contracara de esa fragmentación es la dificultad para que emerjan proyectos políticos que calen en la subjetividad y en las prácticas del día a día de los sujetos, y que sean capaces de 
dar respuestas a experiencias tan diversas. Esa realidad es diversa, y compleja, y optamos trabajar con un sector al que se designa como el de organizaciones y movimientos populares, que cuentan con dos características: luchan contra formas de desposesión, opresión y explotación, y lo hacen con fuerte protagonismo de sujetos pertenecientes a las clases subalternas (Michi et al., 2012). En este sentido, la noción de experiencia es fundamental para nuestro análisis porque en ella -de acuerdo a la tradición que nos inscribimos- se produce el encuentro entre lo consolidado en formas culturales y lo nuevo. Es justamente en esta categoría donde se unifican también esas dos dimensiones centrales señaladas por Marx: la conciencia y la existencia (Williams, 1980; Thompson, 1989a, 1989b). Estas apreciaciones remiten a una perspectiva en la que se considera que para que la realidad cambie hace falta que "muchos" protagonicen esos cambios. En palabras de Michi: "Las formaciones de clase, sus capacidades y su identidad se construyen, tanto en la confrontación social y política (lucha de clases), como en los espacios de autogestión y autogobierno dentro de la misma organización" (2010, pp. 27-28).

En este aspecto la constitución subjetiva es clave porque es lo que permite cuestionar el orden vigente. Sin embargo, tal como nos advierte Gramsci, este proceso de formación crítica y además de intervención activa y consciente en el proceso de la historia del mundo (la praxis), es parte de la formación político-ideológica que surge a partir de y con la acción. Así, otro elemento muy importante a tener en cuenta en nuestro análisis es que todo proyecto de construcción contrahegemónico debiera poseer una praxis pedagógica acorde a la crítica de la dominación que, en cierto sentido, prefigura un nuevo orden social.

Por lo tanto, una de las características destacadas de estos movimientos populares consiste en la prefiguración -o anticipación- de la sociedad futura en la actualidad de su praxis, por medio de acciones autoafirmativas y la construcción de una institucionalidad de nuevo tipo. El concepto de prefiguración lo retomamos del corpus gramsciano y de las relecturas para el campo educativo (Ouviña, 2011). Según esta perspectiva, la disputa por forjar relaciones sociales e instituciones de nuevo tipo comienza en el seno del sistema capitalista aún vigente, que anticipa la sociedad futura en el aquí y el ahora de la construcción territorial.

Otro aspecto importante sobre el cual nos pareció importante reflexionar fue en torno del sujeto campesino que habita la provincia de Mendoza, y la territorialidad que lo circunda, más aún cuando las luchas sociales locales se estructuran a partir su re-apropiación del espacio, o como lo expresa Juan Wahren: “...la presencia del territorio y la cultura de los actores subalternos en los intersticios de las relaciones de dominación, son las que habilitan los procesos autonómicos" (Wahren, 2011). El fenómeno de la territorialización del capital agrario en la Argentina (Hocsman, 2014) ha sido configurado, durante gran parte del siglo XX, principalmente a partir de una matriz de exportación de alimentos acompañada en menor escala por una dinámica de provisión de productos para el mercado interno. Sin embargo, la neoliberalización de las condiciones agrarias en el país sumada a la sojización de la agricultura nacional de las últimas décadas, permiten cartografiar las diferencias de la ruralidad nacional. En los últimas años, mientras por un lado un sector de la pampa húmeda argentina desarrollaba niveles de productividad y acumulación de ganancias prominentes -no exento de la conflictividad social correspondiente-, la otredad rural del oeste, norte, noreste-noroeste y sur del país, ha experimentado alteraciones en sus estructuras agrarias y sistemas de producción y distribución agrícolas. En este segundo cuadro de producción rural, campesinos e indígenas subsisten y ejercen su derecho a la tierra en tiempos de la transnacionalización del capital y el acaparamiento de los territorios.

Bernardo Mançano Fernandes (2005) sostiene que las disputas territoriales expresan una conflictividad intrínseca de clases sociales. Las formas de producción de sujetos y territorios actúan como una modalidad de ocultamiento de ciertas jerarquías sociales y geográficas, que 
garantizan el mantenimiento de la subordinación entre relaciones y territorios dominantes y dominados. Es en los territorios donde las disputas sociales y políticas conforman "rugosidades" en la construcción de los espacios (Santos, 2000). Mendoza es un territorio marcado por el despojo y la apropiación histórica de la tierra indígena, típicamente extendida por toda la Patagonia y el Norte Argentino desde el Siglo XVI en adelante. Sin embargo, su pueblo, en su doble condición de testigo y víctima del proceso colonizador, ha sido un emergente territorial de la construcción social del espacio a través del tiempo (Santos, 2000).

Tanto los trabajadores sin tierra e indígenas en el campo, como aquellos sectores populares que habitan barriadas marginales, unos en zonas de regadío sin acceso a la propiedad de la tierra; otros sin agua ni garantía de derechos básicos, pueblan la geografía provincial dando cuerpo a la movilización que resiste las crisis sociales, ecológicas y sanitarias provocadas por el avance del neoliberalismo en los territorios. Así como en la conformación de las clases y las subjetividades políticas de Nuestra América, el entramado de vinculaciones sociales, políticas y culturales locales se expresan por vía de los conflictos que interpelan las consciencia de los actores colectivos en el desarrollo de sus actividades, prácticas y resistencias -más aun en tiempos de pandemia. El sujeto, en definitiva, "no es, sino que vive siendo y, en sus experiencias de insubordinación, afirma su autovaloración y su confianza en sí mismo y ensancha la brecha personal y colectiva que va abriendo en la pesada losa de la dominación" (Modonesi, 2010, p.10). El carácter político de procesos de subjetivación asentados en relaciones de dominación y de conflicto, permiten reconsiderar la subalternidad de los movimientos sociales latinoamericanos.

\section{¿Cómo? La Educación Popular como camino en la (re)producción de otra cultura}

Nuestra labor se apoya en la experiencia de un conjunto de sujetos/as que en nuestro continente dieron forma a lo que se conoce como "Educación Popular", y que se define como aquella educación que acompaña los procesos organizativos de los sectores populares. En este sentido, consideramos que,

...no puede significar otra cosa que impulsar una acción educativa desde dentro de la misma práctica política liberadora, como una dimensión necesaria de la actividad organizativa de las masas (...) La educación popular, pues, será tal, en la medida que sea -efectiva y prácticamente- un arma que permita a las clases populares asumir organizadamente con lucidez y pasión, su rol de sujetos activos en la construcción de la historia (Jara, 1985, p. 51).

Como cualquier experiencia, la de los movimientos y organizaciones resulta formativa para quienes nos involucramos en ella. $Y$ esa dimensión formativa puede potenciarse, fortalecerse (Michi et al., 2012). En este sentido, nos pareció clave abordar la cultura en clave hegemónica:

La politicidad de la cultura en clave de "hegemonía”. Esto significa colocar la pregunta acerca de las relaciones de poder en el centro de las preocupaciones por los modos en que los grupos sociales organizan simbólicamente la vida en común. Los valores y las creencias, el sentido de las prácticas, las formas de concebir lo propio y lo extraño, lo semejante y lo diferente, y de definir las categorías que procuran ordenar el mapa social son interrogados en su articulación con procesos de construcción, validación o desafío de lo legítimo y lo subalterno, de relaciones de jerarquía o de desigualdad, de mecanismos de inclusión y exclusión. Este punto de partida epistemológico de los Estudios Culturales implica que el poder no es externo a la cultura y, además, implica que este no tiene una esencia propia. En términos ontológicos, el poder no es sino ejercicio y relación (Grimson y Caggiano, 2010, p. 18). 
En tanto ejercicio y relación, el poder se despliega en las aristas de lo dominante y lo marginal, por ello también en el relato de lo rural, lo campesino -ni que decir lo indígena-. Entonces, se puede observar a los sectores sociales analizados, como atravesados por la siguiente condición: se trata de clases subalternas que también son clases populares y se sitúan, al decir de Gramsci, siempre a la defensiva aun cuando se rebelan (Modonesi, 2018). La barbarie tal como se la presenta a modo de ficción -en términos de Piglia- resulta en la región cuyana una persistencia histórica y un modo de salvarse colectivamente: "sin las organizaciones, sería imposible resistir el aislamiento y la pandemia".

Tal como sostiene Modonesi (2018) bien le cabe a Antonio Gramsci no ser un teórico de la subalternidad, sino de la salida de la subalternidad, de la construcción histórica de un sujeto social y político autónomo capaz de disputar la hegemonía; sin embargo, los primeros estudios sobre las culturas populares son producto del impacto de la obra de Gramsci (Alabarces et al., 2007). El intelectual italiano desarrolla el concepto de hegemonía para definir a las clases subalternas como "aquellas dominadas en una relación de poder basada en la hegemonía. Si la alteridad es condición de posibilidad de toda identidad en las clases subalternas esta condición se ve exasperada por la violencia de lo no subalterno entendido como hegemónico" (Alabarces y Añon, 2016, p. 15). Precisamente, la asimilación que Gramsci hace entre clases subalternas y pueblo (Guha, 1997, p. 32) indica las connotaciones de lo subalterno, en tanto:

...masa de la población trabajadora y de los estratos intermedios... no podemos excluir a los sujetos "improductivos", a riesgo de repetir el error del marxismo clásico respecto al modo en que se constituye la subjetividad social. Necesitamos acceder al vasto y siempre cambiante espectro de las masas: campesinos, proletarios, sector formal e informal, subempleados, vendedores ambulantes, gentes al margen de la economía del dinero, lumpen y ex lumpen de todo tipo, niños, desamparados, etcétera (Alabarces y Añon, 2016, p.16)

Así como en Gramsci los grupos sociales se estructuran sobre el desarrollo de las fuerza materiales de producción, en nuestro caso lo subalterno está contenido en lo popular y lo popular en la organización del mundo explotado; las organizaciones populares de Mendoza conforman un tipo de resistencia autónoma frente a las formas de avance del capitalismo y sus pandemias sociales/ sanitarias.

\section{El conocimiento como parte de la praxis}

Entendemos que en el mismo proceso de vida de las organizaciones y movimientos se van produciendo, recuperando y recreando conocimientos.

La noción de praxis, compatible con la propuesta de Ecología de Saberes de Boaventura de Sousa Santos (2009), nos ayuda a comprender cómo se dan estos procesos. Esta categoría fue tomando centralidad en el pensamiento crítico (Gramsci, 1970, 1981; Castoriadis 1999, Freire, 1999; Kosik, 1983). Algunas de las características de la praxis que nos orientan a la hora de comprender la relación que las organizaciones y movimientos pueden tener con el conocimiento son las siguientes: es práctica consciente que integra acción y reflexión o teoría y práctica. Si bien puede haber momentos de predominio de una u otra, siempre están integradas en la misma; el saber previo a la práctica, que a veces se conceptúa como teoría, es siempre fragmentario y provisional; la realidad es dinámica, compleja y multidimensional. A cada momento emergen dimensiones de la realidad que necesitamos conocer. La acción consciente sobre esa realidad es la que genera nuevos conocimientos. En tanto acción sobre una realidad que es multidimensional, se resiste a los límites de las diversas fragmentaciones generadas en la modernidad-colonialidad (Quijano, 
2000; Walsh, 2007; Lander, 2000), especialmente la histórica delimitación de las disciplinas. Además, una realidad que puede ser interpretada desde múltiples epistemologías (De Sousa Santos, 2009); el propósito de la praxis es la transformación de esa realidad.

En este sentido, los conocimientos recuperados, producidos o puestos en juego en la praxis de las organizaciones y movimientos sociales se encarnan para el historiador inglés E. P. Thompson "en tradiciones, sistemas de valores, ideas y formas institucionales" (Thompson, 1989, p. 14). Así, los conocimientos a los que aludimos, y que ocupan un lugar central en los procesos de formación dentro de los movimientos y organizaciones populares, no pueden reducirse entonces a categorías estáticas y dicotómicas como: elaborados vs. populares, universales vs. particulares, científicos vs. sentido común. Entendemos que en el proceso de la propia producción de los movimientos y de su cultura (que, reiteramos, incluye el conocimiento), se van construyendo síntesis (con diversos grados de provisoriedad y de fragmentación) de conocimientos de muy diverso origen, los que siguen en permanente construcción en la praxis cotidiana y son objeto de recuperación, transmisión y recreación constantes. Estos procesos, en tanto anclados en la acción sobre la realidad, imbrican además las múltiples dimensiones de lo humano (intenciones, valores, afectos, razón, corporeidad). Son conocimientos basados en esas epistemologías diversas que fueron negadas por la modernidad eurocéntrica (ausencias) y requieren ser potenciadas (emergencias), siguiendo a Boaventura de Sousa Santos (2009).

A partir de estas consideraciones podemos sostener que el conocimiento universitario no puede ser suficiente para actuar más que como un conocimiento también fragmentario y provisional, lo que no quiere decir que no tenga una considerable valía. Simplemente, no es suficiente y nunca podría ser acabado. En este sentido, procuramos la vinculación con los sujetos sociales colectivos como un diálogo que contribuya a la construcción de un conocimiento múltiple, una ecología de saberes (De Sousa Santos, 2009) que a la vez nos compromete en una praxis compartida que genera nuevo conocimiento en el proceso de transformación de la realidad.

Dicho esto, señalaremos que la hipótesis que sostenemos en este trabajo es que los efectos de la pandemia a escala global y de manera particular en territorio mendocino. Mendoza es una provincia del oeste de Argentina, localizada al este de la cordillera de los Andes. Es una de las principales ciudades del país, y con su aglomerado urbano, denominado Gran Mendoza alcanza una población total que supera el millón de habitantes. Su superficie es de $57 \mathrm{~km}^{2}$, aunque su área metropolitana se extiende $168 \mathrm{~km}^{2}$. Es un principal polo industrial, un punto estratégico fundamental de las relaciones del Mercosur. La actividad económica está vinculada al comercio, la industria de servicios y principalmente la actividad turística en torno a la industria vitivinícola) potencian y explicitan con mayor claridad las construcciones que, desde hace tiempo, vienen gestando en silencio desde la economía social y solidaria, la educación popular, feminista y el protagonismo infantil; las organizaciones y espacios de base en los territorios de los movimientos sociales. Allí emerge un panorama de solidaridad alternativo, pero similar a las experiencias colectivas de resistencias y de supervivencia que caracterizaron los años de la "crisis del 2001". A fines del 2001 se instala el "corralito", medida económica que limita el retiro de efectivo de los depósitos bancarios, afectando a los sectores medios, asimismo, como expresión de la penuria económica de sectores populares se desarrollan saqueos a comercios y supermercados. Se decreta estado de sitio y en respuesta a ello, en los días 19 y 20, se desarrollan "cacerolazos" y masivas movilizaciones que son brutalmente reprimidas con un saldo de 40 muertos. "Que se vayan todos" es una de las consignas que prevalece. Tras estos sucesos renuncia el presidente Fernando De la Rúa y todo su gabinete y se asiste, posteriormente, a la sucesión de cinco mandatarios en sólo diez días. El trueque, las ferias populares y los emprendimientos productivos, entre otros, son la base de los entramados sociales que se fueron fortaleciendo lentamente a lo largo de esos 
años, articulando organizaciones con procesos productivos en diferentes rubros, fortaleciendo e intercambiando "nuevos" saberes en el hacer (Ceceña, 2008).

En lo que sigue entonces, presentaremos una primera sistematización del repertorio de las estrategias y acciones colectivas de los movimientos populares de la provincia de Mendoza y las reflexiones que hacen las mismas organizaciones sobre el impacto de la pandemia en sus territorios. Estas "praxis emergentes" intentaremos expresarlas en clave de saberes, para (re) construir colectivamente una cartografía de las resistencias en nuestra provincia.

\section{METODOLOGÍA}

El 29 de junio de 2020, con el título "Disputas en tiempos de pandemia. Organizaciones y resistencias en contexto de excepción" una gran entrevista colectiva "a cielo abierto" tuvo lugar con una representatividad efectiva de las organizaciones y movimientos populares de la provincia de Mendoza e invitados/as de otras ciudades. La estrategia metodológica cualitativa elegida implicó, tal como señalamos antes, el contacto directo y sincrónico -de manera virtual- entre investigadores/as y organizaciones/movimientos populares, tanto urbanos como rurales.

Las técnicas de construcción de los datos se completaron con el análisis de las declaraciones, manifiestos e intervenciones de esas organizaciones en los primeros meses del avance pandémico en la región.

En esta instancia, sumamente enriquecedora, fue emergiendo un sentido fuertemente compartido entre los y las participantes del encuentro: "sin las organizaciones sociales no hubiese sido posible sostener el aislamiento impuesto por la pandemia". Las problemáticas centrales que emergieron de las distintas intervenciones, las organizamos en torno a cinco ejes-problema:

- Crisis alimentaria

- Violencia hacia las mujeres

- Educación en pandemia

- Higiene y salud

- Situación laboral

Tal como señalamos antes, intentamos aportar con nuestras investigaciones a la teoría crítica en tanto "conjunto de posiciones epistemológicas que entienden, que el conocimiento no puede ser separado de las condiciones ni del contexto de su creación" (Güereca Torres, 2016, p. 32). Dado que la investigación en ciencias sociales implica un proceso de identificación y elección de los hechos relevantes a estudiar, generación de supuestos y preparación de las técnicas de investigación empíricas necesarias, aquí hemos optado por identificar una problemática fundamental como ha sido la pandemia y sus efectos en las clases populares del territorio provincial de Mendoza, con la pretensión de incorporar una mirada visión global y dialéctica al proceso organizativo de los movimientos aquí enunciados.

En tal sentido, se optó por un proceso de investigación-acción participativa llevado a cabo entre los meses de marzo a julio del año 2020. Puntualmente aquí se recoge la palabra de la organizaciones -entendidas como co-investigadores que participan activamente en el desarrollo de la misma (Güereca Torres, 2016)- a partir de conjunto de conversatorios virtuales entre los y las integrantes del Proyecto de Investigación y el grupo de organizaciones aquí enunciadas, a saber: Organización Político-territorial Violeta Parra, La Veleta y la Antena, Frente Popular Darío Santillán-Corriente Nacional (FPDS-CN) Mendoza, Movimiento Popular-La Dignidad (MPLD), Organización EL ARCA, UST- MNCI (Unión de Trabajadores Rurales Sin Tierra - Movimiento Nacional Campesino Indígena / Somos Tierra), Organización Identidad Territorial Malalweche. 
Específicamente en lo que respecta a la producción de conocimientos desde y en los movimientos sociales, los procesos vinculados a la reconstrucción de sus experiencias y sistematización de sus prácticas han formado parte de la preocupación de muchos de ellos. En general, estas experiencias se identifican o relacionan con la tradición de Investigación Acción Participativa (IAP), que surgió en América Latina en la década de los sesenta, en un contexto de ebullición y radicalización política, en oposición crítica a las ideas dominantes en investigación social. La IAP emergió "desde dos vertientes, inicialmente separadas, que luego confluyeron: una pedagógica, asociada al trabajo de Paulo Freire (1970) y otra sociológica, vinculada a Orlando Fals Borda (1961, 1970, 1985 entre muchos otros)" (Torres Carrillo, 2015, pp.11-12). De este modo, las preocupaciones de la IAP se enfocaron en torno a las articulaciones entre acción y conocimiento y relación entre investigadores/as - objeto de conocimiento - sujetos populares, entre otras. Dos ejes claves atravesaron esta corriente. Por un lado, un eje de carácter epistémico según el cual en todos estos procesos debían generarse conocimiento, pero en una perspectiva crítica, reconociendo que la producción de conocimiento no es neutral, siempre responde a la situación y a los intereses de los sujetos que lo producen desde su base social (Fals Borda, 1985, p. 126). Por otro lado, la centralidad del diálogo de saberes en esa construcción colectiva de conocimientos. Tal como lo indica el autor, "la IAP postula que el conocimiento obtenido sobre el terreno y sometido luego a un serio proceso de sistematización para la comprensión cabal de los propios recursos, no pertenece al investigador ni al activista involucrado en las tareas"; y, con la clara preocupación de aportar a través de la producción de saberes al fortalecimiento de organizaciones críticas y autónomas, "forma parte de la praxis de la investigación participativa (no es asunto separado de ella), porque constituye otro elemento de la vivencia colectiva que impulsa las metas de la transformación social” (Fals Borda, 1985, pp. 111-112). Es decir, el objetivo no es “aplicar" teorías surgidas en otros contextos e incluso otros continentes, sino de generar categorías elaboradas colectivamente, que aporten a la comprensión de las propias realidades.

\section{RESULTADOS}

\section{Mapeo de las organizaciones populares en Mendoza}

A partir de la herramienta elegida, se pudo recabar información acerca de las posiciones y estrategias asumidas por las organizaciones, siguiendo las exposiciones de sus integrantes.

\subsection{Crisis alimentaria y falta de insumos para enfrentar la pandemia}

Una de las organizaciones que participó en el conversatorio fue la "Organización Político-territorial Violeta Parra”, barrio La Favorita. Esta organización trabaja en barriadas del gran Mendoza desde la educación popular. Respecto de las problemáticas referidas a la pandemia, sus miembros resaltaron las dificultades que generó el "paso a la virtualidad" en el Bachillerato Popular; espacio educativo que lleva el mismo nombre que la organización. Las múltiples complicaciones de las estudiantes, que en la mayoría de los casos no tenían acceso a internet y el único dispositivo con en el que podían conectarse -en general un teléfono celular conectado con datos a cargo de sus ya complicadas economías- debía estar a disposición de sus hijos e hijas para las tareas de la escuela, que además ellas debían acompañar y sostener. Por otro lado, señalaron el peligro de la falta de agua que los barrios del oeste mendocino padecen cotidianamente. La histórica falta de agua en este contexto, se ha convertido en una carencia mortal. En este sentido se planteó que, aunque repitan hasta el hartazgo la importancia de lavarse las manos, surge un problema fundamental “¿Cómo hacerlo cuando pasan semanas sin que salga una gota de agua?”.

Respecto a la crisis alimentaria, la organización pasó de tener un merendero para los niños y niñas que asistían a los talleres, a organizar un comedor que, al momento del encuentro, 
garantizaban 200 viandas aproximadamente, tres veces por semana, el mismo lo sostenían a través de la autogestión, con donaciones y algunos recursos de la organización. Se planteó que el aislamiento social preventivo y obligatorio (ASPO) en el barrio se transformó en una "sitiada", con un fuerte control policial sobre los/as vecinos del barrio. Respecto a la violencia de género, desde la organización comentaron que estaban armando una guía para actuar en casa, en caso de violencia de género, porque estaban al tanto del crecimiento de esta problemática con el aislamiento.

Por su parte la organización "La Veleta y la Antena” -movimiento que acompaña a niños, niñas y adolescentes que trabajan habitualmente en chacras (cultivo y cosecha de papa, tomate, cebolla, ajo y vides) y también en "Ferias" vendiendo con sus familias, especialmente en el distrito de Ugarteche, Luján de Cuyo-, se resaltó el agravamiento en las condiciones laborales que trajo aparejado el devenir del aislamiento social. La organización actualmente cuenta con un equipo estable de unos 40 niños, niñas y adolescentes trabajadores de entre 8 y 17 años.

Los trabajos en territorio llevados a cabo por los integrantes de esta organización son alternados, dado que la cosecha es estacional durante el verano y la feria funciona los días domingos. Muchos niños, niñas y adolescentes trabajan entre semana cuidando a sus hermanos y hermanas menores para que los/as adultos/as trabajen durante la semana y puedan ir a la escuela. Por otra parte, la población de Ugarteche es migrante, en su mayoría de Bolivia; allí los comedores/merenderos han sido vital para estas familias migrantes. El sostenimiento de estos espacios en pandemia, se ha dado gracias al trabajo de las y los vecinos en al menos 5 de los 23 barrios que conforman el pueblo. Sin embargo, el único comedor activo en la actualidad es el comedor municipal resaltaron los miembros de la organización-. La Veleta y la Antena apoyan a los comedores de los barrios San Expedito, Costa Canal y al propio Comedor Municipal, por la vinculación con el Banco de Alimentos. Además, entregan bolsones mensuales de mercadería (secos) a 20 familias (de las 40 estables) que son parte de la organización.

Uno de los movimientos sociales que participó en esta instancia de investigación-acción fue "Frente Popular Darío Santillán-Corriente Nacional (FPDS-CN) Mendoza”. Organización multisectorial de izquierda autónoma y parte del Movimiento de los Pueblos por un Socialismo Feminista desde Abajo, vinculada al Frente de Organizaciones en Lucha y la organización Violeta Parra. Este espacio colectivo, cuenta con veinte comedores y merenderos alrededor de la provincia, repartidos por los departamentos de Guaymallén, Godoy Cruz, Las Heras, San Rafael, Maipú. La integran también unos veinte grupos productivos que trabajan en textil, herrería, carpintería, panificadoras, en cotillón, reciclados, talleres de cocina, talleres artísticos y apoyo escolar.

Esta organización resaltó que debido a la pandemia se produjo el cierre de las ferias, lo que generó un grave problema para sus integrantes, al provocar la escasa o nula capacidad de ingresos, por verse impedida la comercialización de sus productos. Esto generó un aumento de la desocupación en los barrios y, consecuentemente, multiplicó los participantes en merenderos y comedores, los cuales se duplicaron y hasta triplicaron, en busca de su plato de comida diario. Al momento del conversatorio el FPDS registraba entre 40 y 45 mil raciones de alimentos mensuales, con modalidad de entrega de viandas para evitar aglomeraciones de personas y así disminuir los contagios de COVID-19. Por otro lado, se remarcó que la autogestión de los elementos de higiene y seguridad, tales como los tapabocas elaborados por las propias trabajadoras textiles, que fueron destinados a quienes están abocados a las labores en los merenderos y comedores. La desinfección de los espacios comunitarios también es una tarea que ha cobrado particular importancia. En la mayoría de los merenderos se agregaron días de comedor. El aumento de demanda de alimentos y de viandas diarias, tiene como contracara la escasez de recursos en los comedores: "hay mucha 
gente y mucha necesidad, y el hambre no se hace esperar...pero es muy difícil mantener ese nivel de trabajo por falta de insumos", argumentaban los integrantes del FPDS.

Por otra parte, esta organización resaltó que fue a través de mucho trabajo y donaciones que se fueron sorteando la escasez de alimentos. Claudia, del FPDS comentó:

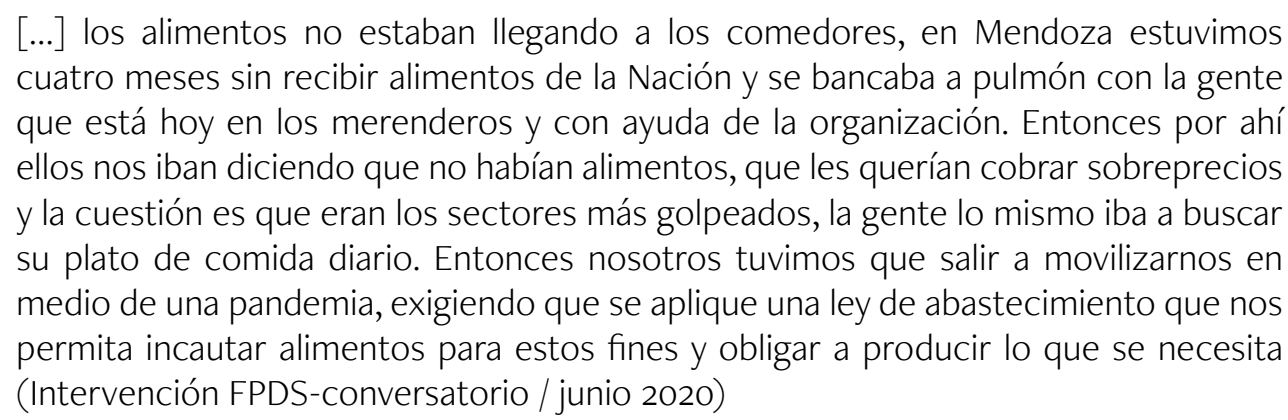

Entre las estrategias para afrontar la crisis económica y sanitaria destacan el valor de la información y la comunicación sobre los cuidados propios y con los otros/as que hay que tener en estos tiempos. Dada la desinformación que circula, el compartir conocimientos y estrategias entre todos fue el modo más efectivo que encontraron para ello. Al no recibir ayuda del gobierno en este aspecto "con los recursos que teníamos hacíamos lo que íbamos pudiendo". Se elaboraron cartillas para prevenir el coronavirus y el dengue, también para cuidarse de los abusos de la policía y la violencia machista. Se hicieron talleres de salud comunitarios en los cuales también se repartían guantes, folletería y un termómetro para los espacios comunitarios.

En la misma dirección se inscribe la intervención del Movimiento Popular-La Dignidad (MPDignidad). Se trata de una organización nacional vinculada a los espacios ideológicos reconocidos como izquierda popular. Este movimiento participa de la Confederación de Trabajadores de la Economía Popular CTEP, que a su vez aglutina el sindicato UTEP (Unión de Trabajadores de la Economía Popular). El MP-Dignidad tiene como base de formación la construcción del poder popular con una integración suburbana desde la economía popular, salud comunitaria y feminismo popular, al respecto de las preguntas formuladas, manifestaron lo siguiente:

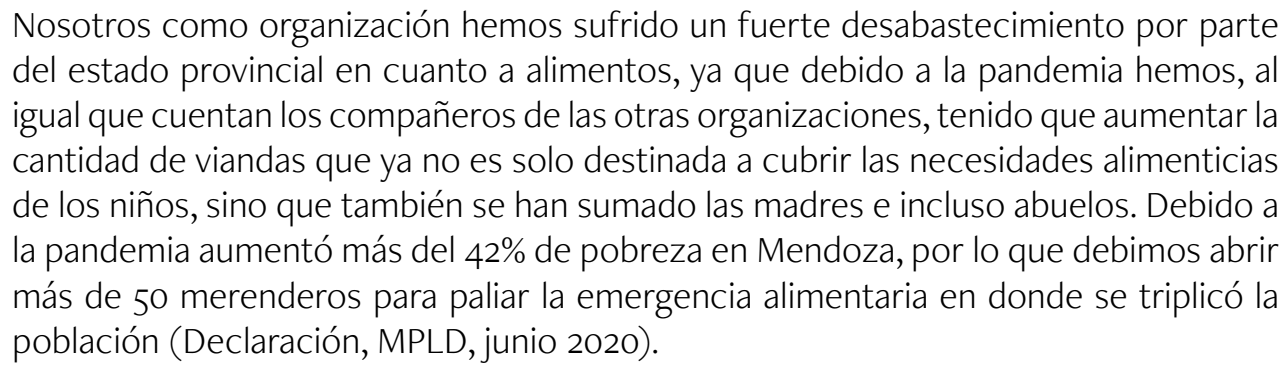

Además de tener que enfrentar con muy pocos recursos las cuestiones sanitarias, falta de agua y productos de higiene en las comunidades en las que están insertos.

La falta de agua ha sido un tema recurrente y sigue siendo, para esta organización la pandemia los encontró enfrentados al gobierno provincial, por el fuerte abandono en la obligación de cumplimentar, la aplicación de políticas públicas para el sector popular, tales como el Ingreso Federal de Emergencia (IFE) (Intervención MP-La Dignidad).

Otro tanto resaltó El ARCA, organización de 15 años que reúne a productores y consumidores de Mendoza y Argentina, articulados en torno del concepto de "comunidad prosumidora", cuyo ADN es el trueque: 
La pandemia para nosotros ha sido un desafío muy grande que hemos tenido que enfrentar como organización, hemos tenido un fuerte impacto en la generación de trabajo que veníamos llevando adelante, tuvimos que organizarnos estratégicamente para que no cayeran las ventas en la producción de envasados y elaboración textil. Muchas compañeras que cosen en sus casas se quedaron sin el trabajo que hacían, pero por suerte supimos reinventarnos y ahora ellas están cosiendo ropa descartable, indumentaria sanitaria (barbijos, camisolines, etc.) porque hemos tenido que aumentar las producciones e incluso organizarnos en la distribución de los alimentos (Intervención de integrantes de El Arca, junio 2020)

Argumentaron que esta situación les ha permitido incrementar las ventas, en tanto se ha podido organizar en la distribución de la producción de alimentos y generar más lazos con los consumidores y nuevos productores.

\subsection{Violencia hacia las mujeres, higiene y precariedad laboral}

Respecto a la violencia contra las mujeres, la organización La Veleta y la Antena trabaja principalmente con la violencia -de fuerte impronta patriarcal (adultocéntrica)- contra los niños, niñas y adolescentes. Ésta ha sido una de las problemáticas centrales en este tiempo para $L a$ Veleta, dado que las consultas dan como resultado el avance de los "problemas de la convivencia" según las propias palabras de los niños y las niñas. Durante la cuarentena han tenido dos hechos de trascendencia por violencia contra una niña de 6 años y una mujer de 30 años (esta última, tuvo cobertura mediática). Las acciones que llevó a cabo la organización fueron el aumento de contactos telefónicos y fortalecimiento de los vínculos con las familias de los niños, niñas y adolescentes trabajadores que forman parte de la organización. Además, elaboraron cartillas de trabajo sobre educación sexual integral que abordan concretamente la violencia sexual contra niños, niñas y adolescentes. Con el apoyo de la Asociación Ecuménica de Cuyo, imprimieron 1200 cartillas para 6 escuelas primarias de la sección 17 (Ugarteche, Carrizal y Agrelo, distritos de Luján de Cuyo) con quienes han fortalecido una red de trabajo en este periodo. Las cartillas las entregaban las directoras de los colegios con los bolsones de mercadería y las actividades de las cartillas las recibían las docentes en ejercicio.

Además, el espacio del encuentro provincial de niñas y adolescentes, que han conformado una comisión organizadora junto a otras organizaciones que trabajan con niñez, estaban elaborando, al momento del conversatorio, una radio para que pudieran seguir en contacto. Las integrantes de esta organización resaltaron el trabajo en conjunto con las bibliotecas Jesús Nazareno, David Blanco, Álvarez Condarco y la organización Pibas Autoconvocadas, para realizar spots de difusión vía redes sociales para la concientización sobre los derechos y las infancias. Sobre esto último, la organización resaltó la importancia de la reactivación en los acompañamientos a embarazos forzados en niñas y adolescentes durante el periodo de aislamiento. Desde los años 2017 y 2018 esa se ha tornado una tarea novedosa para la organización (que ya realizaba acompañamientos locales a niñas víctimas de violencia sexual) y se extendió a la provincia (a excepción de zona sur). En este periodo pandémico, esto ha vuelto a interpelar el trabajo de esta organización. Así manifestaron, al momento del conversatorio, que se encontraban con dos acompañamientos de manera telefónica y con la presentación de un proyecto para la erradicación de la violencia de género en la infancia para su aprobación ante la Secretaría Nacional de Niñez, Adolescencia y Familia (SENAF).

Por último, con los hechos acontecidos en Ugarteche, se estában proponiendo un grupo de debate sobre violencia sexual, tema que no se lograba instalar en la comunidad y que el asalto violento a una mujer adulta frente a sus hijos e hijas, puso de relieve. La Veleta encontró opciones para acompañar el aislamiento, conversando con las familias, identificando los problemas y en red con 
las escuelas, con otras organizaciones sociales. Se resaltó en la instancia del conversatorio que para familias quechua parlantes, que no escriben el castellano o que tienen muchos hijos e hijas, copiar tareas desde un teléfono, les impedía realizarlas. Para ello dispusieron el recurso de un proyecto de investigación del Colegio de Trabajo Social, para la compra de tonner y resmas de papel:

Una vecina ofreció su computadora y la Biblioteca Popular Héroes de Malvinas (hoy inactiva) su impresora. Desde allí durante los últimos dos meses, se imprimen las tareas de 42 familias y unos 56 niños, niñas y adolescentes, de primaria 1-726 Estación José Francisco Ugarteche y otras aledañas, así como algunas estudiantes secundarias y de la universidad. Las dificultades de aprendizaje, desde nuestra organización demandan apoyo escolar, pero cuando regrese la actividad, en acuerdo con las escuelas (Declaración La Veleta y la Antena, junio 2020)

Respecto a la situación en Higiene y Salud del territorio donde está inserta, la organización retoma los aportes de la Dra. Florencia Linardelli en un informe realizado a CONICET. Dicen al respecto: "El riesgo mayor de la población en su conjunto se vincula con el acceso a los servicios de salud". Se estima que la cantidad de profesionales es particularmente baja frente a la cantidad de población a cargo. Finalmente, señalan que la infraestructura de los centros de salud (CAPS Nº 36 y N$^{\circ} 39$ ) es limitada para el abordaje de la pandemia en curso y ejemplifican:

Los centros de salud de la zona funcionan en casas de barrio adaptadas y las condiciones edilicias no sirven para esta coyuntura. Tenemos una sola puerta de ingreso y no hay manera de separar a la población que espera ser atendida. Por ahora hemos decidido que esperen afuera. Pero en los próximos meses, con el frío y las condiciones climáticas del invierno local, no podemos pedirles a las mujeres con sus hijos recién nacidos que permanezcan a la intemperie. En ese momento las personas sanas se van a cruzar con quienes estén infectadas en recintos muy pequeños que son las salas de espera. ¿Cómo haces si sos un solo profesional que tiene que atender a todos? Porque no podés mandar de vuelta a los recién nacidos a sus casas. Sos una sola persona que tiene una sola puerta y tenés que atender todas las situaciones. Más allá de la contingencia epidemiológica, los problemas de dotación de personal y las condiciones edilicias son estructurales en esta zona. La necesidad es de siempre. (Declaración La Veleta y la Antena, junio 2020)

Concretamente Ugarteche cuenta con un centro de salud para el primer nivel de atención y una población de 30 mil habitantes. El acceso al agua en el distrito es limitado, y el aumento poblacional lo hace aún más escaso. Han recibido alcohol en gel, que el municipio entregó en los domicilios. Pero las condiciones de salubridad y las condiciones habitacionales (que impiden el abrigo, son permeables), la ausencia de baños, de cloacas y de agua, refleja las condicionantes en el cuidado. Es una gran preocupación el acceso al agua.

Los problemas específicos que han surgido por las medidas de aislamiento son diversos. La situación más reiterada entre los/as informantes son las dificultades para proveer de alimentos a las familias. Estas dificultades, de acuerdo con los/as informantes, se vinculan con la disminución del ingreso monetario de los grupos familiares; con el cierre de comercios dispuesto a nivel provincial a las 18 horas, mientras que la jornada de trabajo agrícola culmina a las 19 horas; y con una situación específica de desabastecimiento local, puesto que es una zona alejada de grandes centros urbanos (el más próximo queda a 20 kilómetros). El cierre de la feria de la localidad es destacado como un motivo adicional que complica las posibilidades de compra de alimentos. En algunos hogares no se dispone de heladera para preservar los alimentos, razón que empuja a las familias (en general a los trabajadores estacionales y mujeres solas con niños/as) a realizar compras diariamente. 
En el caso de las familias, la falta de trabajo (mal Ilamado informal, cuando en Latinoamérica, no es la excepción sino la regla), limita los ingresos familiares. O donde antes trabajaban todos, hoy sólo lo hace el varón adulto. Finalmente, respecto a la situación de las infancias, para la organización es fundamental visibilizar la preocupación que tienen niños y niñas respecto al contagio, a la provisión de alimentos, a la falta de trabajo, a recibir buen trato durante el aislamiento, pero también al tener apoyo es muy importante. Y lo han planteado en primera persona. Generar estrategias de acompañamiento y tranquilidad en las viviendas, ha sido una línea de acción que debió “inventarse” y “rápido". En este sentido, acciones como "tirar la Veleta por la ventana” y repartir todos los libros y los juegos de mesa para el entretenimiento y fundamentalmente para sostener los vínculos y los afectos políticos. Desde la organización les parece fundamental difundir estas voces, y visibilizar finalmente que el trabajo de niños, niñas y adolescentes, de cuidado de hermanos/as, de ancianos/as convivientes, de familiares enfermos/as, el trabajo doméstico y trabajo escolar que realizan a diario (reproductivo), tanto como el trabajo productivo que han dejado de realizar (venta, chacra, etc) que hizo evidente la disminución del ingreso familiar, y puso sobre la mesa (tanto como el escándalo en la terminal de ómnibus por los trabajadores y las trabajadoras del norte) que el trabajo de niños, niñas y adolescentes existe y debe ser parte de la política sobre niñez.

A la par de la pandemia de coronavirus hubo un importante incremento de la violencia de género, especialmente intradoméstica. Sobre esto el FPDS remarcó:

Nos organizamos mejor para acompañar a las mujeres en situación de violencia, en los últimos meses se incrementó mucho la violencia de género, ya venía, y se incrementó más, hubo cincuenta muertes, hoy día se agregaron dos más, fue muy duro y es muy duro para las personas que están viviendo este tipo de violencia. Entonces nos organizamos para acompañar a estas mujeres y luchamos para que sus situaciones de vida cambien. Pudimos conseguir colchones y frazadas porque tuvieron que salir de sus lugares sin nada, entonces nos pusimos en campaña para conseguir colchones y frazadas para poder ayudarlas. También estamos en la lucha para ayudar a nuestras compañeras trans y travestis que están en la misma situación (Declaración FPDS, junio 2020).

Otro problema que detectan desde el FPDS es el aumento de la violencia policial:

muchas veces los compañeros iban a trabajar a los merenderos y se encontraban un móvil policial y los paraban y los hacían volver, o te volvés o te saco una multa o te llevo preso, entonces los compañeros no podían llegar a su lugar de trabajo a asistir a la gente". Estas problemáticas (pandemia, salud, desocupación, violencias de género y policial) iban acompañadas de situaciones de precarización estructural en los barrios. Una deuda histórica que tiene el gobierno en muchas zonas de la provincia es el acceso al agua potable: "el otro tema era no tener agua, acá en los barrios de Mendoza, por ejemplo, el Campo Papa, Colonia Segovia, La Favorita no tienen agua, entonces es imposible poder trabajar. En San Rafael no hay agua entonces se tiene que estar comprando, se tiene que juntar plata para comprar agua porque si no el merendero no puede funcionar (Declaración FPDS, junio 2020).

\subsection{Educación en pandemia y trabajo desde la economía social}

Garantizar el derecho a la educación en tiempos de aislamiento y clases virtuales frente a la realidad que se describe ha sido particularmente dificultoso: 
Por ahí hay mamás que no saben, no tienen recursos para poder ayudar a sus hijos, el tema de la conexión a internet porque no hay wi-fi, porque no tenemos datos, porque hay un solo teléfono, dificulta mucho. Entonces esto va llevando a los problemas familiares, por ahí el marido que trató mal a la mujer porque no le supo enseñar a su hijo, hay muchas mamás que son analfabetas, entonces se va generando un clima de violencia donde la mujer se ve muy afectada, muy exigida. Se implementó en esos lugares el apoyo escolar con los protocolos de cuidado, y turnos para ir atendiendo a los chicos, irles ayudando para que esto no sea de discordia en la familia y se pueda dar una respuesta colectiva a este problema (Declaración FPDS, junio 2020)

Desde el FPDS se planteó que ha habido una muy mala gestión gubernamental de la pandemia, destinando fondos públicos para otras cosas y no para lo que es prioritario para el pueblo: "nosotros venimos pidiendo la recomposición salarial y la apertura de nuevos cupos para los planes de empleo. Con la pandemia pedimos la universalización del IFE, que resultó completamente insuficiente. Hay mucha desocupación y el gobierno está haciendo oídos sordos a esto". Además, hicieron, durante el conversatorio, una especial mención a aquellas mujeres que han estado trabajando pese a toda adversidad en las ollas populares: "queremos reconocer a las compañeras que están bancando las ollas populares, porque es un esfuerzo muy grande, muy grande...hoy estas compañeras están poniendo todo...para poder estar ayudando a nuestros vecinos, familiares y compañeros para poder enfrentar esta pandemia".

Otras de las organizaciones activas en toda la provincia de Mendoza y los territorios rurales del país es la Unión de Trabajadores Rurales Sin Tierra, integrante del Movimiento Nacional Campesino Indígena / Somos Tierra (UST-MNCI). Esta organización campesina de unos 18 años de existencia desarrolla tareas de arraigo rural, comercialización, producción agroecológica, disputas por la tierra y procesos de educación popular, por medio del proyecto político pedagógico del CEFICTierra (Centro de Educación, Formación e Investigación Campesina) en el cual se articula la Escuela Campesina de Agroecología, la Tecnicatura en Economía Social y Desarrollo Local y otras instancias de formación política. Sobre las preguntas formuladas, apuntaron lo siguiente:

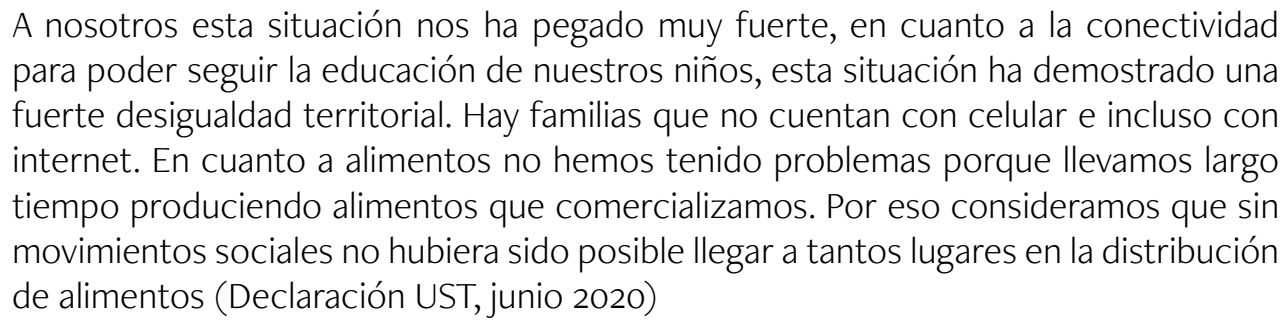

Finalmente, la “Organización Identidad Territorial Malalweche”, también formó parte de dichos conversatorios. Tras 13 años de un proceso territorial propio, esta organización reivindica el derecho a la producción caprina y la identidad indígena en el territorio provincial. Articulan con el sector de la economía popular y social agregando valor a la producción de alimentos saludables. Sostienen la Cooperativa Killen en todo el territorio rural y urbano de Malargüe. Participan de la Federación de Trabajadores de la Economía Social (FETRAES)

Para nuestra comunidad la pandemia y crisis nos ha permitido fortalecer lazos, nosotros no tenemos como cosmovisión la asistencia. Debido a este contexto tuvimos que abrir esa cosmovisión en la asistencia alimentaria a la comunidad en general llevando bolsones de verdura y de carne (Declaración Malalweche, junio 2020)

Se argumentó que la preocupación más fuerte que tienen sobre la "post-pandemia" es el avance del extractivismo, dado que su territorio se encuentra en zonas de resistencia a la explotación 
de fracking (sistema de extracción petrolera mediante la modalidad de fractura hidráulica). Se sostiene una posición de resistencia territorial, "como comunidad acordamos, en general, en que es una pandemia que se inicia con Macri (presidente argentino) y que hemos tenido que reconfigurar nuevas estrategias para poder hacer frente a la emergencia alimentaria".

\section{CONCLUSIÓN}

\section{Cartografiar resistencias. Algunas reflexiones finales}

Tal como señalamos al comienzo de este trabajo, el mundo desde el que aquí se enuncia está atravesando una crisis abrupta y profunda. En tal sentido, el lugar habitado por las resistencias, producto de la pandemia del Covid-19, ha dejado expuesta las precariedades existentes y el rol fundamental de las organizaciones para afrontarlas.

A partir de lo analizado, resaltan algunas consideraciones que es necesario apuntar. Por un lado, acerca de la construcción de saberes con y desde los movimientos sociales, a lo largo de esta experiencia surgieron una serie de interrogantes y preocupaciones que se han ido constituyendo en áreas de indagación e investigación en nuestro equipo. Nuestras miradas se centraron en las potencialidades de las organizaciones para impactar sobre la realidad social y también sobre las subjetividades. Con relación al primer aspecto, fuimos centrando nuestra mirada en las formas reivindicativas que adoptan las organizaciones y los mecanismos de disputa, así como su vinculación con las esferas del Estado. Con respecto al segundo elemento, nos interesamos por los procesos de organización interna, los mecanismos de toma de decisiones y los procesos de formación que han ido construyendo. Actualmente nuestra preocupación central, a la que dedicamos nuestros esfuerzos por producir conocimiento, está dada por el modo en que las organizaciones populares construyen subjetividad, es decir, la manera en que intervienen dotando de sentido sus propias experiencias, aspectos que serán motivo de otro trabajo

Por otro lado, en relación a los resultados presentados en este trabajo nos parece importante señalar que -tal como mencionaron las organizaciones participantes- los problemas estructurales no empezaron en marzo del 2020 ni hace cuatro años. El hacinamiento, la falta de servicios básicos como el agua y la angustia del desempleo y la informalidad se arrastran desde hace décadas y los ejes problemáticos que identificamos en las experiencias de las organizaciones que participaron del conversatorio, son prueba de ello. La violencia económica tampoco es nueva, Mendoza registra un 41,1\% de trabajo informal, sin contar la subocupación o el desempleo. Se estima que la mayor parte de los trabajadores que se encuentran en una situación de informalidad laboral, se quedaron sin ingresos con la pandemia. En estas condiciones el aislamiento impuesto por la pandemia impide el ingreso económico de muchas familias y las empuja al hambre.

Una de las caras más crudas del aislamiento lo viven las mujeres y las infancias. Las primeras, a las que se les ha impuesto históricamente las tareas de cuidado y de sostenimiento de los hogares, y que -a pesar de los avances- siguen estando casi exclusivamente a su cargo. Ahora no sólo tienen que hacer tareas diversas y precarias para pensar cómo sostener la economía familiar, sino que el trabajo invisibilizado se ha multiplicado y se suma el trabajo de ser maestras de sus hijos/as en medio de la incertidumbre y la falta de recursos. A esto se le agrega, en muchos casos, que el aislamiento sea junto con su agresor.

Otro aspecto que advirtieron las organizaciones territoriales de la periferia urbana mendocina, fue el despliegue de fuerzas represivas para "cuidar" a la población. Frente a esto nos parece necesario reflexionar en dos sentidos: por un lado, recordar que éstas son las mismas fuerzas de seguridad que organismos de derechos humanos y anti represivos han venido denunciando 
durante décadas por su accionar criminal (expresado en torturas, detenciones arbitrarias, casos de gatillo fácil, entre otras). Por otro lado, no desatender cuál es el papel de la policía en los barrios. La narcopolicía, las zonas liberadas, las alianzas con el poder político, la complicidad con femicidas y violentos, la revictimización y maltrato a las denunciantes, entre miles de tareas que realiza esta fuerza; no tienen nada tienen que ver con tareas de "cuidado".

Ante este panorama, las organizaciones apuestan a la solidaridad. Emergen nuevamente propuestas de comedores y merenderos, circulan viejos recetarios empolvados que se usaron en las más tristes postales del 2001. Las redes se extienden y crecen día a día. Los/as vecinos se involucran y participan. Además, las diversas experiencias organizativas y productivas que llevan adelante los movimientos sociales, dan cuenta de su capacidad para generar su propio trabajo, producir alimentos de calidad y demostrar que existen otras formas de asumir la organización, cumplir con las necesidades de su entorno, así como también, la producción, la distribución y comercialización de alimentos, de maneras más justas y cooperativas. Dicho proceso, lejos de ser estimulado o promovido, parece ser una respuesta a una histórica situación de vulnerabilidad, desigualdad social (económica, productiva, cultural, de género, territorial, comercial, etc.) y exclusión, generadora de injusticia social. La exclusión es la cara de la injusticia social, responsable no sólo de un reparto desigual de los bienes, las tareas, el poder, sino que también del reconocimiento y rol asignado dentro de la sociedad.

Esta característica se vio acrecentada en el actual contexto de pandemia (COVID-19), dentro del cual, los movimientos sociales en el marco de la economía social/popular contribuyeron a la seguridad alimentaria y nutricional local, posicionándose como un actor central en la provisión de alimentos básicos, constituyéndose como uno de los estereotipos del trabajador esencial. Más aún, en un marco en donde la pandemia exacerbó la crisis económica y limitó el proceso de producción, circulación y consumo, estos movimientos organizados lograron reinventarse, (adaptarse) produciendo y profundizando estrategias alternativas de producción y abastecimiento alimentario, formando redes y formas de comercialización, que si bien, se vienen gestando hace años con otras organizaciones -generando trabajo y proveyendo alimento en cantidad, calidad y mayor accesibilidad- en este contexto se fortalecieron.

El arraigo territorial de estos movimientos ha permitido hacer frente a la crisis con estrategias enmarcadas en la solidaridad, la autogestión y la horizontalidad, todo lo cual vuelve su estudio ineludible para pensar la post-pandemia. Los trabajadores sin tierra e indígenas en el campo, como aquellos sectores populares que habitan barriadas marginales, unos en zonas de regadío sin acceso a la propiedad de la tierra; otros sin agua ni garantía de derechos básicos, pueblan la geografía provincial dando cuerpo a la movilización que resiste las crisis sociales, ecológicas y sanitarias provocadas por el avance del neoliberalismo en los territorios. El carácter político de procesos de subjetivación asentados en relaciones de dominación y de conflicto, permiten reconsiderar la subalternidad de los movimientos sociales en territorio argentino y cuyano.

\section{REFERENCIAS}

Alabarces, P. y Añón, V. (2016). Subalternidad, pos-decolonialidad y cultura popular: nuevas navegaciones en tiempos nacional-populares, Versión. Estudios de Comunicación y Política, (37), 13-22.

Alabarces, P. (2007). Un destino sudamericano. La invención de los estudios sobre cultura popular en la Argentina. En P. Alabarces y M. Rodríguez (eds.), Resistencias y mediaciones. Estudios sobre cultura popular. (pp. 261-280). Paidós.

Castoriadis, C. (1999). La institución imaginaria de la sociedad. Vol. 1 y 2. Tusquets editores.

Ceceña, A. (2008). De los saberes de la emancipación y de la dominación, Buenos Aires, 1a ed. CLACSO 
CEDES (Centro de Estudio de Estado y Sociedad) (2020). Estudio Tiaria. Primer avance de resultados. CEDES.

CEPAL (Comisión Económica para América Latina y el Caribe) (2020). Dimensionar los efectos del COVID-19 para pensar en la reactivación, Informe Especial COVID-19. CEPAL https://repositorio.cepal.org/ bitstream/handle/11362/45445/1/S2000286_es.pdf

De Sousa Santos, B. (2001). Los nuevos movimientos sociales, Revista OSAL, 5.

De Sousa Santos, B. (2009). Una epistemología desde el Sur. CLACSO y Siglo XXI.

Fals Borda, O. (1985) Conocimiento y Saber Popular. Siglo XXI

Fernandes, B. M. (2005) Movimentos socioterritoriais e movimentos socioespaciais: Contribuição teórica para uma leitura geográfica dos movimentos sociais. Revista OSAL. 6(16). https://doi.org/10.47946/ rnera.voi6.1460

Freire, P. (1991). Pedagogía del oprimido. Siglo XXI

Gramsci, A. (1975). El materialismo histórico y la filosofía de Benedetto Croce. Juan Pablo.

Gramsci, A. (1981). La alternativa pedagógica. Fontamara.

Gramsci, A. (1988). Textos de los cuadernos de la cárcel 1929-1931. En A. Gramsci, Antología de Antonio Gramsci. Siglo XXI

Gramsci, A. (1998). La política y el Estado Moderno. Fontamara

Grimson, A. y Canggiano, S. (2010). Respuestas a un Cuestionario: posiciones y situaciones. En N. Richards (editora) En torno a los estudios culturales locales, trayectorias y disputas (pp. 17-30) Ed. Arcis - CLACSO.

Grupo de Trabajo CLACSO Crisis y economía mundial (2020) Nuestra América XXI. Desafíos y alternativas 48. CLACSO.

Güereca Torres, R. (2016). Guia para la investigacion culitativa: etnografia, estudio de caso e historia de vida. Universidad Autónoma Metropolitana.

Guha, R. (1997). Prefacio a los Estudios de la Subalternidad. En S. Rivera Cusicanqui y R. Barragán, Debates poscoloniales: una introducción a los Estudios de la Subalternidad, traducción de Raquel Gutiérrez, Alison Spedding, Ana Rebeca Prada y Silvia Rivera Cusicanqui, (pp. 23-24) Sephis/Aruwiyri,

Hocsman, L. (2014). Tierra, capital y producción agroalimentaria: despojo y resistencias en Argentina. En G. Almeyra, L. Concheiro Bórquez, J. Márcio Mendes Pereira y C. Walter Porto-Gonçalves (Coord.) Capitalismo: tierra y poder en América Latina (1982-2012) Argentina, Brasil, Chile, Paraguay, Uruguay Volumen I. (pp. 17-57) Ediciones Continente.

Jefatura de Gabinete de ministros Argentina (2020) Informe 125. Honorable Senado de la Nación Argentina. https://www.argentina.gob.ar/sites/default/files/informe_125_.pdf

Jara, O. (2012). La sistematización de experiencias, práctica y teoría para otros mundos posibles. San José: CEP Alforja/CEAAL/ Oxfam Intermon

Jara, O. (1984). Los desafíos de la educación popular. Tarea

Jara, O. (1981). Educación Popular: la dimensión educativa de la acción política. Panamá, Ceaspa.

Kessler, G., y Benza, G. (2020). elmpactará la crisis de covid-19 en la agenda social de América Latina? Nueva Sociedad. https://cutt.ly/sQdLvtV

Kosik, K. (1983). Dialéctica de lo Concreto. Estudio sobre los problemas del hombre y el mundo. Editorial Grijalbo S.A.

Lander, E. (2000). ¿Conocimiento para que? ¿Conocimiento para quién? Reflexiones sobre la universidad y la geopolítica de los saberes hegemónicos Rev. Venez. de Econ. y Ciencias Sociales, 6(2), 53-72.

Lander, E. (Comp.) 2000. La colonialidad del saber:eurocentrismo y ciencias sociales. Perspectivas latinoamericanas. CLACSO.

Martínez Pineda, M. C. y Guachetá Gutiérrez, E. (2020) Educar para la emancipación. Hacia una praxis crítica del sur. Bogotá. CLACSO, Universidad Pedagógica Nacional.

Michi, N. (2010). Movimientos campesinos y educación. Estudio sobre el Movimento de Trabalhadores Rurais Sem Terra y Movimiento Campesino de Santiago del EsteroVC. El Colectivo.

Michi, N., Di Matteo, J., y Vila, D. (2012) Movimientos populares y procesos formativos. Revista Polifonías, $1(1), 22-41$

Ministerio de Desarrollo Productivo (2020) Informe de Panorama Productivo. Ministerio de Desarrollo 
Productivo. https://cutt.ly/MQdLSbL

Modonesi, M. (2010). Subalternidad, Antagonismo, Autonomía: Marxismo y subjetivación política. Prometeo CLACSO.

Modonesi, M. (2018). Consideraciones sobre el concepto gramsciano de clases subalternas. Memoria, Revista de Política y Cultura, (265). 61-66. https://revistamemoria.mx/?p=1952

Molina, M. (2019) Saberes y territorio la disputa de sentido desde los movimientos sociales (Mendoza, 2001-2018). Recuperado de: https://bdigital.uncu.edu.ar/fichas.php?idobjeto=14745

Núñez Hurtado, C. (1998) La Revolución Ética. IMDEC.

Ouviña, H. (2011). La política prefigurativa en el joven Gramsci. Una aproximación a la teoría y práctica de la educación futura. En F. Hillert, H. Ouviña, L. Rigal y D. Suárez. (comps.). Gramsci y la educación: pedagogía de la praxis y políticas culturales en América Latina (pp. 141-172). Noveduc.

Ouviña, H. (2015). Educación en movimiento y praxis prefigurativa. Una lectura gramsciana de los proyectos pedagógico-políticos impulsados por los movimientos populares latinoamericanos. En F. Hillert, H. Ouviña, L. Rigal y D. Suárez, Pedagogías críticas en América Latina: experiencias alternativas de educación popular. Noveduc.

Quijano, A. (2000) Colonialidad del poder, eurocentrismo y América Latina. En L. Edgardo (Comp.) La colonialidad del saber: eurocentrismo y ciencias sociales. Perspectivas latinoamericanas. CLACSO.

Santos, M. (2000). Por uma outra globalização: do pensamento único a consciência universal. Editora Record.

Soto, O., y Caisson, S. (2020). Periferias, Estado y movilización popular. Apuntes de coyuntura en tiempos de pandemia en Argentina. Revista Ichan Tecolot/ CIESAS-CONACYT México. https://cutt.ly/gQdXgp9

Tapia, L. (2008). Movimientos sociales, movimientos societales los no lugares de la política. En L. Tapia (2008) Política Salvaje. CLACSO, Muela del Diablo.

Torres Carrillo, A. (2011) Educación Popular, trayectoria y actualidad. Universidad Bolivariana de Venezuela.

Torres, A. (2014) Producción de conocimiento desde la investigación crítica. Nómadas, 40.

Thompson, E.P. (1989a). La Formación de la Clase Obrera en Inglaterra. Crítica.

Thompson, E.P. (1989b). Tradición, revuelta y conciencia de clase. Crítica.

Wahren, J. (2011). Territorios Insurgentes: La dimensión territorial en los movimientos sociales de América Latina. [Ponencia] IX Jornadas de Sociología. Universidad de Buenos Aires, Buenos Aires.

Walsh, C. (2007) ¿Son posibles unas ciencias sociales/culturales otras? Reflexiones en torno a las epistemologías decoloniales. NÓMADAS, 26.

Williams, R. (1980). Marxismo y literatura. Península.

\section{AUTORES}

Milagros Molina. Doctora en Ciencias Sociales por Facultad de Ciencias Sociales de la Universidad de Buenos Aires, Argentina. Magíster en Estudios Latinoamericanos con orientación en Cultura y Comunicación por la Facultad de Ciencias Políticas y Sociales, Universidad Nacional de Cuyo. Docente, investigadora y extensionista.

Mariana Polti. Estudiante de grado de la carrera de Sociología por la Universidad Nacional de Cuyo.

Oscar Soto. Doctorando en Ciencias Sociales en la FCPyS-UNCuyo. Magister en Estudios Latinoamericanos

(FCPyS-UNCuyo). Licenciado en Ciencia Política y Administración Pública (FCPyS-UNCuyo). Docente en la cátedra Teoría Política de la Licenciatura en Trabajo Social (FCPyS). Actualmente es becario de CONICET.

\section{Conflicto de intereses}

Los autores informan que no existe conflicto de interés posible.

\section{Financiamiento}

No existió asistencia financiera de partes externas al presente artículo.

\section{Agradecimientos}

Los autores agradecen a las organizaciones y movimientos populares de Mendoza y Argentina por sumarse al trabajo colaborativo y militante del Proyecto de Investigación SIIP: Saberes y territorios. La disputa de sentidos desde los Movimientos sociales (UNCuyo) 